\title{
Quiet Sun coronal heating: Analyzing large scale magnetic structures driven by different small-scale uniform sources
}

\author{
O. Podladchikova, T. Dudok de Wit, V. Krasnoselskikh, and B. Lefebvre
}

LPCE/CNRS UMR 6115, 3A Av. de la Recherche Scientifique, 45071 Orléans, France

Received 29 August 2001 / Accepted 7 November 2001

\begin{abstract}
Recent measurements of quiet Sun heating events by Krucker \& Benz (1998) give strong support to Parker's (1988) hypothesis that small-scale dissipative events make the main contribution to quiet heating. Moreover, combining their observations with the analysis by Priest et al. (2000), it can be concluded that the sources driving these dissipative events are also small-scale sources, typically of the order of (or smaller than) $2000 \mathrm{~km}$ and below the resolution of modern instruments. Thus the question arises of how these small scale events participate in the larger-scale observable phenomena, and how the information about small scales can be extracted from observations. This problem is treated in the framework of a simple phenomenological model introduced in Krasnoselskikh et al. (2002), which allows one to switch between various small-scale sources and dissipative processes. The large-scale structure of the magnetic field is studied by means of Singular Value Decomposition (SVD) and a derived entropy, techniques which are readily applicable to experimental data.
\end{abstract}

Key words. Sun: corona - Sun: magnetic fields

\section{Why small-scale sources?}

The anomalously high temperature of the solar corona is still a puzzling problem of solar physics, in spite of the considerable theoretical and experimental efforts involved for a long time (e.g. Priest et al. 2000; Einaudi \& Velli 1994). Since the energy release in the largest heating events (flares and microflares) does not supply enough power to heat the corona, the statistical behavior of the smaller-scale and less energetic but much more frequent events may be the key to the problem, as was conjectured some time ago by Parker (1988).

Recently, Krucker \& Benz (1998) reported an important result that supports Parker's hypothesis. Using Yohkoh/SXT observations, and assuming that the flaring region has a constant height, they found that in the energy range $10^{24}-10^{26}$ ergs, the energy probability density follows a power law with an exponent of about -2.59 . Such an exponent below -2 suggests that heating takes place on small scales, whereas an exponent greater than -2 would suggest a prevalent role played by large-scale phenomena. The conclusion of Krucker \& Benz was confirmed by Parnell \& Jupp (2000), who estimated the exponent to be between -2 and -2.1 by making use of TRACE data and by supposing that the height is proportional to the square root of the area. Mitra \& Benz (2000) discussed the same observations as Krucker \& Benz by supposing a

Send offprint requests to: O. Podladchikova, e-mail: epodlad@cnrs-orleans.fr height variation similar to that used by Parnell \& Jupp, and showed that the exponent, although larger, is still below -2 .

Making use of multi-wavelength analysis, Benz \& Krucker (1999) also showed that energy release mechanisms are similar in large scale loops and in the faintest observable events. They further noticed that the heating events occur not only on the boundaries of the magnetic network but within the cells too. Priest et al. (1998), by comparing observations with model predictions for plasma heating in the magnetic loop with distributed energy sources, have found that the thermoconductivity along the magnetic field lines due to electrons is high enough and concluded that the heating is quasi-homogeneous along the magnetic loop. These results support the hypothesis that the heating mechanism that is not located in the vicinity of the footpoints but is instead spread over the arc. It then follows that the characteristic spatial scale of the magnetic field loops that supply the dissipated magnetic field may be of the same order of magnitude as the characteristic dissipation scale. We may thus conclude that not only the dissipative process, but also the energy sources have small characteristic length.

However, Aschwanden with co-authors (2000) analyzed TRACE observations of the nano-flares in the energy range from $10^{24}$ to $10^{26}$ ergs in two wavelengths $171 \AA$ and $195 \AA$. They have shown that "EUV nano-flares represent miniature versions of larger flares observedon soft X-rays 
(SXR) and hard X-rays (HXR) scaled to lower temperatures $\left(T_{\mathrm{e}}<2 \mathrm{MK}\right)$, lower densities $\left(n_{\mathrm{e}}<10^{9} \mathrm{~cm}^{-3}\right)$ and somewhat smaller spatial scales $(l \approx 2-20 \mathrm{Mm}) . "$ They showed that the cooling time is defined by the radiative cooling, but the conductive cooling timescale is about an order of magnitude shorter suggesting repetitive heating cycles. They suggested that the heating mainly takes place around the footpoints, implicitly suggesting that the scale of sources can be even smaller than the scale of dissipation sites. They presented the spatial distribution of nano-flares that seem to be quite homogeneous (see Fig. 1 of their paper). They suggest that the heating takes place on even smaller scales than nanoflares. We use in our model the quite similar idea that the sources should be distributed homogeneously in space.

It is therefore important to discuss the role and the properties of sources and dissipative processes in the framework of simplified models. Such an approach allows us to study the correspondence between large-scale magnetic field properties and the characteristics of the smallscale (eventually smaller than the experimental resolution) sources and dissipative events. In this work we shall investigate the possibility of getting information about small-scale magnetic energy sources by making use only of the large-scale magnetic field, as would be the case with experimental data. To do so, we shall consider a phenomenological model and apply to it different statistical tests.

A phenomenological model allowing for different types of sources and physical dissipation mechanisms was recently proposed in Krasnoselskikh et al. (2002). The results concerning the temporal statistics of the total dissipated energy are briefly recalled in the next section. To investigate the spatial properties of the magnetic fields and the dissipative events, statistical tests are needed, which can be applied both to simulation and to experimental data. Tools such as the magnetic field entropy and extraction of the most energetic and large-scale spatial/temporal eigenmodes by means of Singular Value Decomposition (SVD) are described in Sect. 3. Their application to our model and their ability to discriminate between various sources and dissipative mechanisms are discussed in Sect. 4, and the final section proposes a review and a critical discussion of the results.

\section{Small-scale driving and dissipation}

Various phenomenological models of flare-like events and cooperative phenomena in the corona have been considered in the literature (e.g. Lu \& Hamilton 1991; Vlahos et al. 1995; Georgoulis et al. 2001), mostly relying on the notion of Self-Organized Criticality (Jensen 1998). Such models usually exhibit infinite-range spatial correlations, and due to the tenuosity and localization of the driving do not provide an appropriate framework for our purpose.

Instead, we shall use the model introduced in Krasnoselskikh et al. (2002) and Podladchikova et al. (1999) which allows for a driving more distributed in space and dissipative processes relevant to heating studies. As usual, the model represents a simplification of the magnetohydrodynamic induction equation

$\frac{\partial \boldsymbol{B}}{\partial t}=\nabla \times(\boldsymbol{u} \times \boldsymbol{B})+$ dissipative term

The turbulent photospheric convection in some sense randomizes the first term on the right hand side, which can be replaced by various source terms with specified statistical properties. The dissipative terms may take into account different effects such as normal and anomalous resistivity or magnetic reconnection, which in general depend on the current density and magnetic field configuration. Their meaning and the differences between the two in this context are discussed at length in Krasnoselskikh et al. (2002).

A cellular automata model combining the direct solution of the MHD equations in a 3D geometry with the local magnetic field dissipation using large-scale energy sources associated with the vector potential $\boldsymbol{A}$ was studied by Isliker et al. $(2000,2001)$. The authors have shown that large scale variations of the vector potential in the framework of their model can give rise to the formation of the smaller-scale currents. The dissipation in their model is similar to "anomalous resistivity dissipation" that we study here.

The model is two-dimensional, the magnetic field being perpendicular to the grid, with periodic boundary conditions. A discrete description of the magnetic field in terms of cells is proposed, while the currents are computed from

$\left(\begin{array}{c}j_{x} \\ j_{y}\end{array}\right)=\frac{1}{\delta}\left(\begin{array}{c}B(x, y)-B(x, y+\delta) \\ B(x+\delta, y)-B(x, y)\end{array}\right)$

where $\delta$ is cell length ( $\delta=1$ in the following). The currents can be considered as propagating on the border between the cells, and satisfies Kirchoff's law at each node.

As discussed in the introduction, one may suppose that the source terms that represent the magnetic energy injection and the dissipative processes have comparable spatial scales. Therefore, the source terms that mimic the magnetic energy injection from the turbulent photosphere are assumed to have a vanishing time average; they act independently on each cell, at each time step. Three types of sources with different statistical properties are considered:

- Random sources. The simplest source consists of random variables $\delta B$ from the set $\{-1,0,1\}$, which act individually on each cell. This source can be made dipolar by dividing the grid into two parts: random numbers from the same set, but with opposite signs are fed into each pair of cells.

- A chaotic source. Turbulence is certainly not a completely stochastic process, and some of its aspects are enlightened by deterministic models. In each cell the source evolves according to

$$
\delta B_{n+1}=1-2\left(\delta B_{n}\right)^{2},
$$




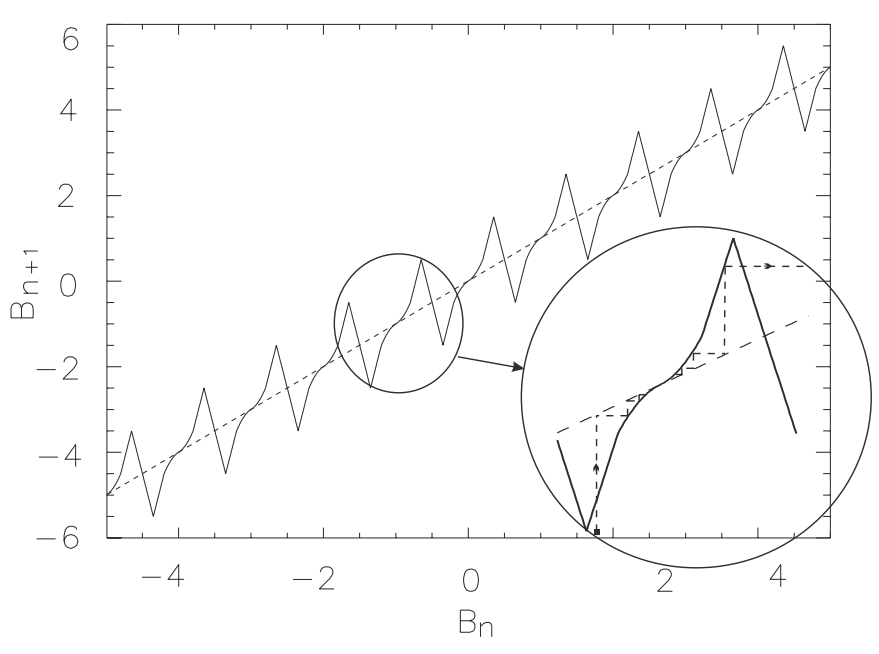

Fig. 1. Graphical representation of the Geisel map (solid line). The fixed points of the map correspond to the intersections of the graph with the straight line $B_{n+1}=B_{n}$ (dashed line).

where $\delta B \in[-1 ; 1]$ and the subscript ${ }_{n}$ denotes the time step. This so-called Ulam map is closely related to the logistic map. Both are well known for their chaotic dynamics.

- Geisel map source. The map introduced by Geisel \& Thomae (1984), hereafter called a Geisel map, provides another example of a source with chaotic dynamics

$B_{n+1}=f\left(B_{n}\right)$.

Because of its marginally stable fixed points, this map may generate anomalous subdiffusion, since

$\left\langle B^{2}\right\rangle \propto t^{\alpha}, \quad \alpha<1$.

It is generally expected that magnetic field lines in a turbulent plasma exhibit a subdiffusive behavior, which is, however, more complex than described above.

We stress again that the sources act independently on each cell, and are updated at each time step. Dissipation is therefore the only mechanism by which neighboring cells can interact.

Dissipation allows for the conversion of magnetic energy into particle acceleration and thermal energy, and in our model provides the coupling between the magnetic field elements. Dissipative processes are most important where a current sheet carrying strong current density has formed. Neglecting resistivity, which is small in the corona, one is left with various instabilities of magnetic field configurations that can cause dissipation. We consider two of them:

- Anomalous resistivity, which arises from the development of certain instabilities such as modified Buneman instability when the electric current exceeds a certain threshold in collisionless plasma. In our model the currents are simply annihilated whenever they exceed a certain threshold,

$|j| \geq j_{\max }$
- Reconnection, for which we require in addition the magnetic field to have an $X$-point configuration. Hence, the following two conditions should be satisfied simultaneously:

$$
\begin{aligned}
|j|=\left|B-B^{\prime}\right| & \geq j_{\max }, \\
B \cdot B^{\prime} & <0 .
\end{aligned}
$$

Because of this new condition, currents may exist that largely exceed the threshold $j_{\text {max }}$.

The main difference between these two processes is that reconnection represents a change in equilibrium, from one topology (here a $X$-point) to another, whereas anomalous resistivity does not require any particular topology and thus may also act on the interior of cells and not only at boundaries. Another important difference is that anomalous resistivity provides Joule-like heating, while reconnection yields accelerated outgoing flows and thus may be associated with non-thermal radiation.

When the current is annihilated, the values $B$ and $B^{\prime}$ of the magnetic field in neighboring cells are replaced by $1 / 2\left(B+B^{\prime}\right)$, so the magnetic energy that is dissipated in a single event becomes (with $\mu_{0}=1$ )

$\Delta E=\frac{1}{4}\left(B-B^{\prime}\right)^{2}=\frac{1}{4} j^{2} \gtrsim \frac{1}{4} j_{\max }^{2}$.

The procedure for modeling the current dissipation is the same regardless of the dissipation mechanism. At each time step, the currents satisfying the dissipation criterion are dissipated until all of them are subcritical (or have the same sign in the case of reconnection). Then, we proceed to the next time step and switch on the source. Indeed, dissipative processes are supposed to be faster than the driving terms. The total dissipated energy is calculated as the sum over all the dissipated currents for the considered time step.

In Podladchikova et al. (1999) and Krasnoselskikh et al. (2002), the influence of the dissipative processes and source terms on the statistical properties of the dissipated energy were studied. The dissipation was found to have a significant influence on the statistics of dissipated energy. Indeed the reconnection mechanism was shown to yield the strongest deviation from a Gaussian distribution in the large energies. However, the probability density of the dissipated energy was shown to be rather insensitive to the nature of the magnetic field sources. In the present paper, we would like to further explore the dependence of the statistical properties of the large-scale magnetic field upon the physical characteristics of the source and dissipation processes. This will be done in the framework of our model. Our objective therefore is to study an inverse problem: how do the large-scale properties of the magnetic field allow us to characterize the sources and the dissipative processes? To do so, we shall use various measures of spatial complexity. 


\section{Characterization of spatial complexity}

Spatial complexity can be characterized in many different ways (e.g. Grassberger 1986). Linear properties are traditionally studied by considering the time averaged spatial correlation function

$C(\boldsymbol{r})=\langle B(\boldsymbol{x}, t) B(\boldsymbol{x}+\boldsymbol{r}, t)\rangle_{\boldsymbol{x}, t} /\left\langle B(\boldsymbol{x}, t)^{2}\right\rangle_{\boldsymbol{x}, t}$,

where the average is carried out over different positions and times (or events). We have computed the characteristic decay length of this correlation function for various sources, dissipation mechanisms, and thresholds.

A different approach, which is commonly used in image processing, is based on the Singular Value Decomposition (SVD) or Karhunen-Loève Transform, see Golub \& van Loan (1996). For each time step, the bivariate magnetic field intensity $B(x, y)$ can be viewed as $2 \mathrm{D}$ image. We decompose this image into a set of separable spatial modes

$B(x, y)=\sum_{k=1}^{N} \mu_{k} f_{k}(x) g_{k}^{*}(y)$.

By making these modes orthogonal $\left\langle f_{k} f_{l}^{*}\right\rangle=\left\langle g_{k} g_{l}^{*}\right\rangle=$ $\delta_{k, l}$, the decomposition becomes unique. The weights $\mu_{k}$ of these modes, also called singular values, are conventionally sorted in decreasing order, and are invariant with respect to all orthogonal transformations of the matrix $B(x, y)$. In our case, the number $N$ of modes is equal to the spatial grid size.

A key property of the SVD is that it captures largescale structures in heavily weighted modes, whereas patterns that are little correlated in space are deferred to modes with small weights. The distribution of the singular values is therefore indicative of the spatial disorder: a flat distribution means that there is no characteristic spatial scale and hence, the magnetic field should not show largescale patterns. Conversely, a peaked distribution suggests that there are coherent structures (Dudok de Wit 1995). It must be stressed that this approach is, like the previous one, based on second order moments only, since the spatial modes and their singular values issue from the eigenstructure of the spatial correlation matrix of the magnetic field.

From the SVD modes of the 2D magnetic field, one can define a measure of spatial complexity, which is called the SVD entropy (Aubry et al. 1991). Let $E_{k}=\mu_{k}^{2} / \sum_{i} \mu_{i}^{2}$ be the fractional amount of energy which is contained in the $k$ th mode. The SVD entropy can then be defined as the limit

$H=-\lim _{N \rightarrow \infty} \frac{1}{\log N} \sum_{k=1}^{N} E_{k} \log E_{k}$.

The maximum value $H=1$ is reached when spatial disorder is maximum, that is when $E_{k}=1 / N$ for all $k$. $H=0$ means that all the variance is contained in a single mode. Note that Aubry et al. (1991) also introduced a purely temporal and a spatio-temporal entropy, but in this paper we shall focus on the spatial entropy only.

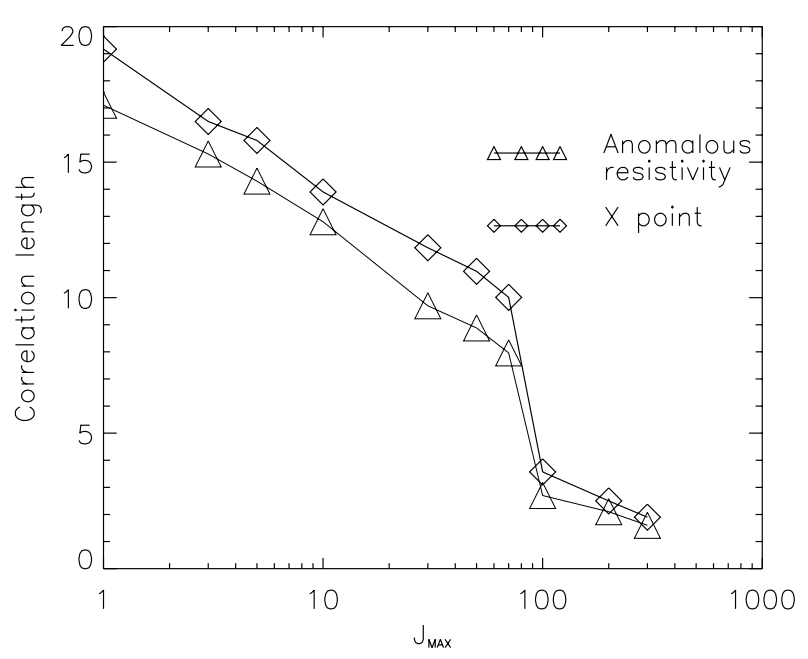

Fig. 2. Dependence of the correlation length on the threshold of dissipation, for both dissipation rules. Anomalous resistivity is marked by triangles, reconnection by squares.

In practice, it is rarely necessary to take the limit $N \rightarrow \infty$ in Eq. (5). Let $H_{M}$ be the SVD entropy as computed from a subset of finite size $M \times M$ (with $M \leq N$ )

$H_{M}=-\frac{1}{\log M} \sum_{k=1}^{M} E_{k} \log E_{k}$.

This quantity shall converge fast enough toward its asymptotic value. For large enough $M, H_{M}$ is thus an intensive quantity (independent of the subsystem size $M$ ) and thus should rather be called an entropy-per-cell.

The SVD can also be used as a linear filter to extract large scale patterns from a background with small-scale fluctuations. To do so, one should perform the SVD and then in Eq. (4) sum over the strongest modes only, to obtain a filtered magnetic field. There is obviously some arbitrariness involved in the identification of what we call strong modes, but the process can be automated by using robust selection criteria, see for example Dudok de Wit (1995).

Notice that in contrast to several other studies, we shall not use the fractal dimension (more exactly, the Haussdorf-Besicovitch dimension) of the magnetic field as an additional measure of spatial complexity. Indeed, we found that in most cases, the differences observed between the different sources and/or dissipation mechanisms was too small relative to the numerous uncertainties that are inherent to the estimation of such a quantity. We note, though, that the 2D magnetic field was generally found to have fractal properties, with a dimension $D=1.5-1.7$.

\section{Spatial complexity and properties of the source and dissipation}

\subsection{Spatial correlations}

The different measures of spatial complexity described above were applied to the 2D magnetic field, after 

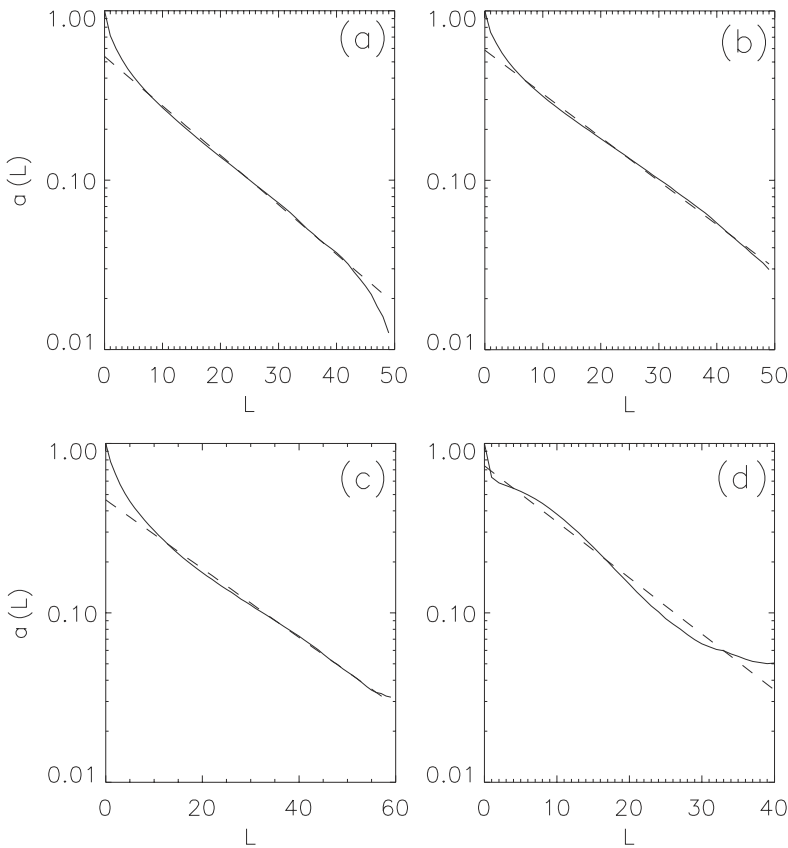

Fig. 3. Averaged spatial correlation coefficient of the magnetic field, with semilogarithmic axes (solid lines). Dotted lines represent the best fit by an exponential function. The results are obtained for random and subdiffusive sources with a $200 \times 200$ grid size and a dissipation threshold $j_{\max }=1$. a) Random source, anomalous resistivity dissipation, correlation length $L \approx 17 ; \mathbf{b})$ random source, reconnection dissipation, correlation length $L \approx 19 ; \mathbf{c})$ subdiffusive source, anomalous resistivity dissipation, correlation length $L \approx 23$; d) subdiffusive source, reconnection dissipation.

initial transients had died out. In the following, we consider time averages.

The spatial correlation function was estimated using Eq. (3). For the small grid sizes, of the order of $30 \times 30$, the correlation function decays as a power-law. As shown in Krasnoselskikh et al. (2002), the probability density of the total dissipated energy in this case also decays as a power-law. This apparent indication for self-organized critical behavior, however, is a mere artifact of the small grid size, since it disappears with larger grids. Indeed, for grid sizes of about $100 \times 100$ and beyond, the correlation functions decay almost exponentially, while the dissipated energy exhibits a quasi-Gaussian distribution.

From the exponentially decaying correlation functions, we define the correlation length $L$ as

$C(r)=\exp (-r / L)$

We found $L$ to remain almost constant as soon as the grid size exceeds about $200 \times 200$. In that case, $L$ is much smaller than the grid size. It is therefore legitimate to expect the results not to depend significantly on the grid size or on the boundary conditions. In the remainder of this paper, we shall only present results for $400 \times 400$ grids. A small threshold $\left(j_{\max }=1\right)$ will be used, which is of the order of $\sqrt{\left\langle\delta B^{2}\right\rangle}$.
As shown in Fig. 2, for a random magnetic field source the average correlation length $L$ is larger for reconnection type dissipation than for anomalous resistivity. This may be explained by the presence of supercritical currents $j>j_{\text {max }}$. Moreover, in both cases the correlation lengths are decreasing functions of $j_{\max }$ (see Fig. 2). We do not find significant differences in the functional dependence of the correlation function or in the correlation length when changing the processes. The single exception is the Geisel map source with reconnection, for which the correlation function decays neither exponentially nor as a power-law, see Fig. 3d. No accurate correlation length can be inferred in that case.

The main result here is that we cannot distinguish between different processes solely on the basis of the correlation length. This is in contrast with the marked differences one observes when visualizing the magnetic fields with different sources, see for example Figs. $4 \mathrm{~b}$ and $5 \mathrm{~b}$. Thus, alternative indicators are needed.

\subsection{Singular values and coherent spatial modes}

As discussed in the previous section, the Singular Value Decomposition provides an orthogonal decomposition which allows us to extract coherent patterns that may possibly exist in the bivariate magnetic field.

The distribution of singular values generally reveals a few large-amplitude modes, followed by a long tail of weak modes, see Fig. 6. Such a distribution suggests that the salient features of the bi-dimensional wavefield are captured by a few modes only. Indeed, a long tail is indicative of fluctuations that are randomly distributed in space. For instance, the most energetic mode $\left(f_{1}(x)\right.$ in the notation of Eq. (4)) for a Geisel source with dissipation by reconnection, clearly reveals a large-scale coherent structure (Fig. 7).

The fact that the most heavily weighted modes correspond to large-scale magnetic field structures, can also be seen by comparing Figs. 4 and 5 . In both figures, the subfigures b) and c) compare the original magnetic field, and the field that has been reconstructed using 20 only of the strongest modes. It appears that the strongest modes capture the large-scale coherent structure of the magnetic field.

This analysis, however, only provides a decomposition of the magnetic field at a given time. No information is obtained about the lifetime of these structures, which is a crucial quantity. It appears, however, that the heavily weighted modes persist for long times, as compared to the original magnetic field. This can be seen by comparing the filtered magnetic field at two instants separated by 2000 time steps (Figs. 5c and d). One can actually see in Figs. $5 \mathrm{a}-\mathrm{c}$ how these structures grow from an initially disordered state.

Thus the coherent structures extracted by SVD have a long lifetime and produce a slow decay of the temporal autocorrelation function. While small-scale structures 

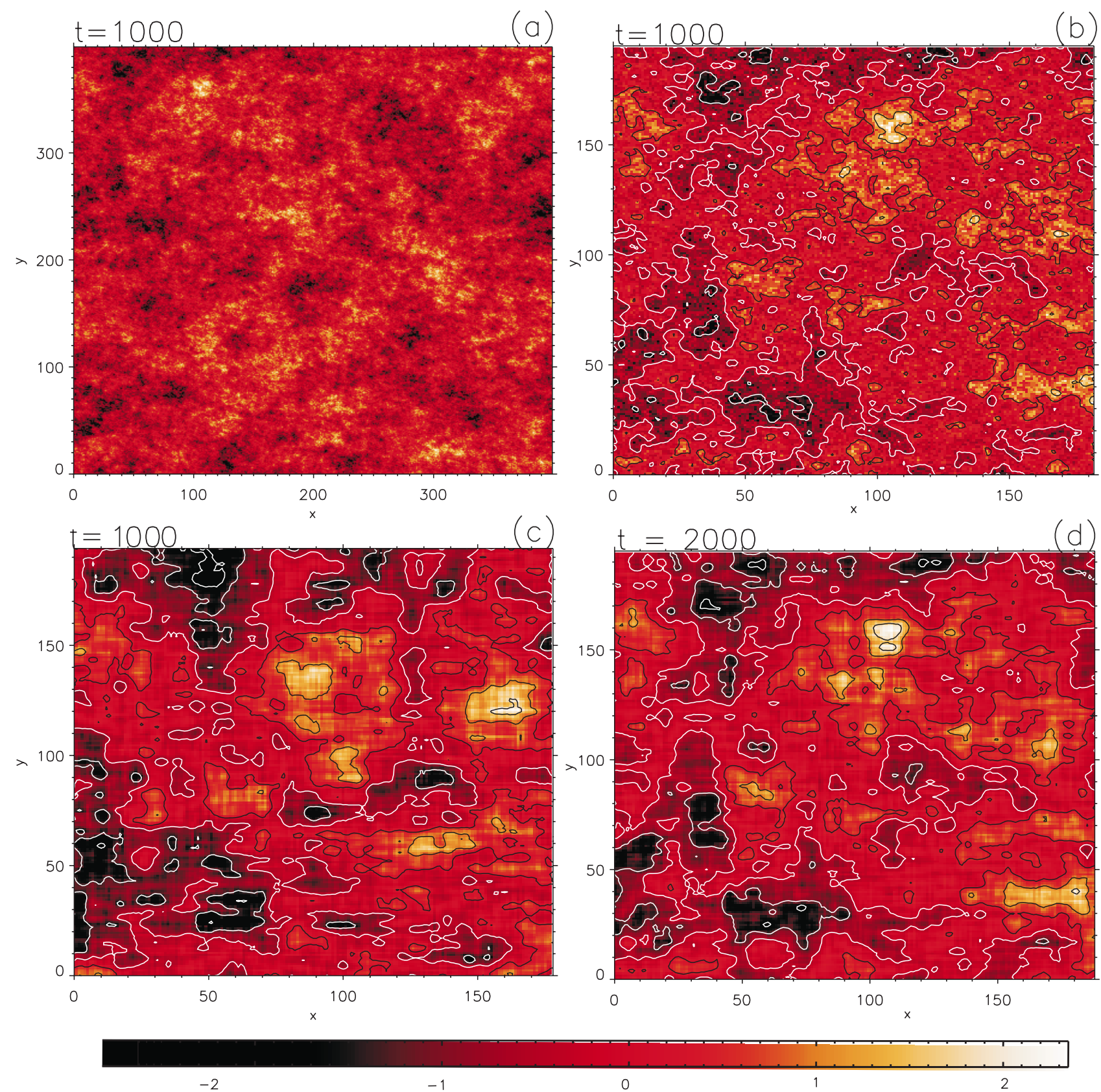

0

$\mathrm{B}_{2}$

Fig. 4. Excerpt of the magnetic field obtained for a random source with reconnection, with $j_{\max }=1$. a) Magnetic field at $t=1000 ; \mathbf{b})$ zoom of the preceding image; c) same zoom as in $\mathbf{b}$ ), but with the wavefield reconstructed using 20 only of the strongest SVD modes, out of 400; d) same zoom as c), but at a later time $t=2000$. The SVD entropies at $t=1000$ and $t=2000$ are respectively $H=0.79$ and $H=0.81$.

rapidly appear and disappear, the large-scale ones evolve slowly. In that sense, they are truly coherent structures.

\subsection{Magnetic field entropy}

Quantitatively, the degree of coherence of the magnetic field can be measured by the spatial entropy (or more exactly entropy-per-cell) defined by Eq. (5) from the singular values. This definition involves a limit $N \rightarrow \infty$, but in practice, for large enough grid sizes, it can be checked that the quantity defined in Eq. (6), computed for a $M \times M$ subset of $B$, converges toward a well-defined limit as $M$ increases. Computing this entropy $H_{M}$ for increasing $M$, we obtain the curves displayed in Fig. 9, which show that the entropy already converges for matrix sizes of about $100 \times 100$. It seems that the convergence is even faster for the subdiffusive source than for the random source. Typically, $H_{M}$ converges when the subsystem size approximately reaches the size of coherent structures. We may thus conclude that the entropy $H$ is fairly independent of 


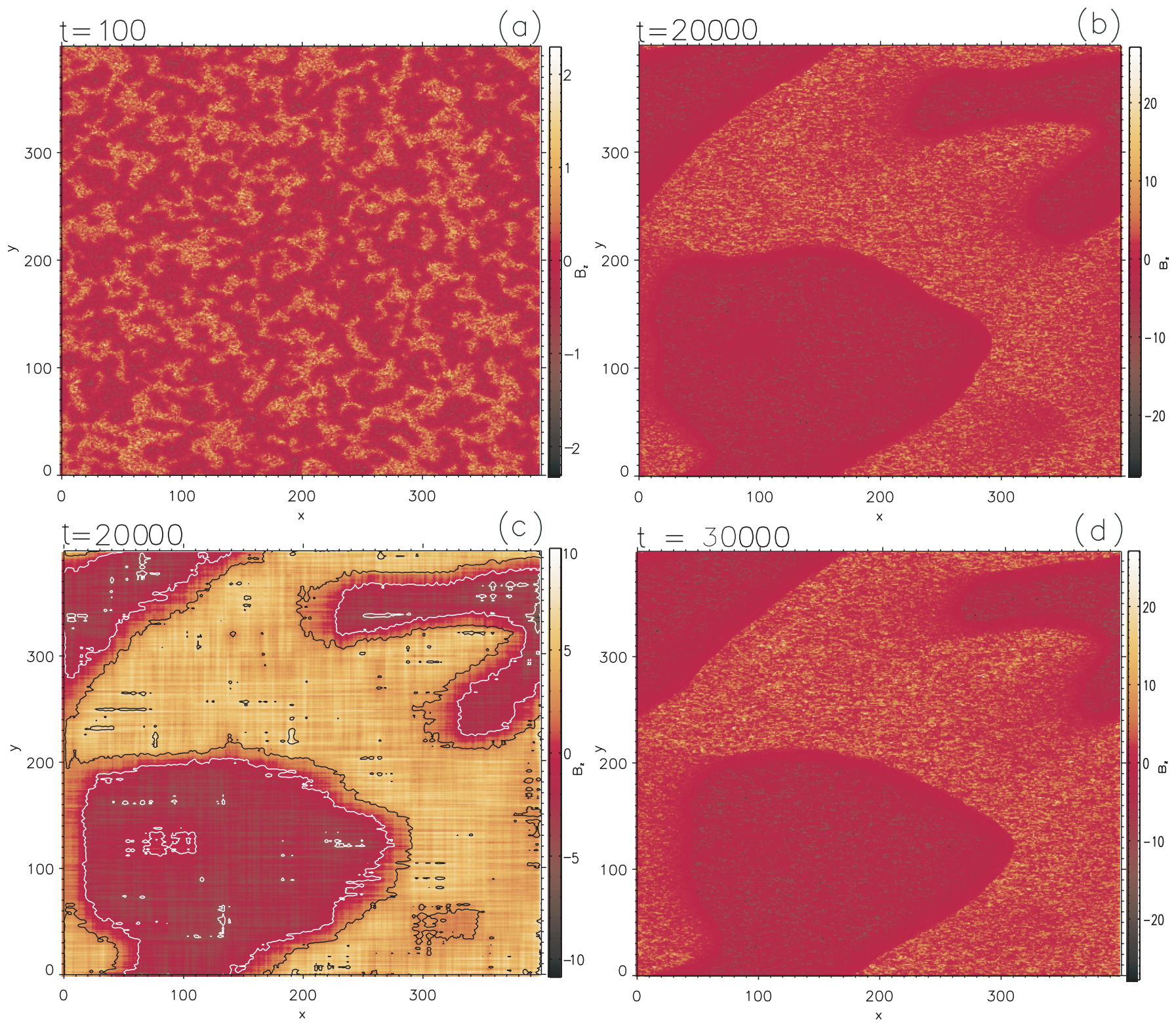

Fig. 5. Excerpt of the magnetic field obtained for a subdiffusive source with reconnection, with $j_{\max }=1$. a) Magnetic field in a transient state, at $t=100 ; \mathbf{b})$ magnetic field in a stationary state, at $t=20000$; $\mathbf{c}$ ) same as in $\mathbf{b}$ ), but with the wavefield reconstructed using 20 only of the strongest SVD modes, out of 400; d) magnetic field at a later time, $t=30000$. The SVD entropies at $t=100$ and $t=20000$ and $t=30000$ are respectively $H=0.73, H=0.51$ and $H=0.53$.

Table 1. Variation of the entropy in time, for the subdiffusive source and reconnection (see also Fig. 5).

\begin{tabular}{r|rrrr}
\hline \hline$t$ & 100 & 500 & 20000 & 30000 \\
$H$ & 0.73 & 0.69 & 0.51 & 0.527 \\
\hline \hline
\end{tabular}

the grid size, provided that the grid size exceeds a threshold value of about $100 \times 100$.

The entropy has a monotonous decay in time and converges toward a finite value in the steady state (see Table 1), indicating the simultaneous decrease of spatial complexity and the formation of slowly evolving largescale magnetic field structures.
Table 2. Entropy in the steady state for various source types, and dissipation by reconnection.

\begin{tabular}{cc}
\hline \hline source type & $H$ \\
random & 0.8 \\
Ulam & 0.78 \\
Geisel & 0.53 \\
\hline \hline
\end{tabular}

The major result here is that the value toward which the entropy converges in time exhibits significant differences when the different sources are used, as summarized in Table 2. 


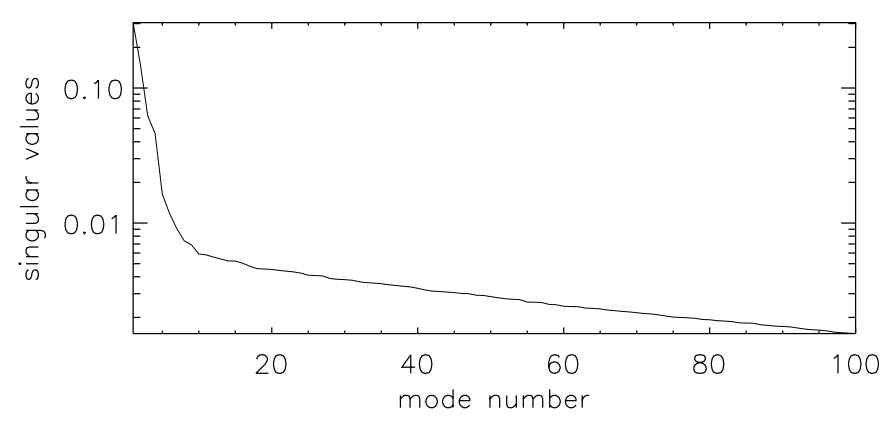

Fig. 6. Distribution of the singular values associated with a $400 \times 400$ magnetic field matrix. Only the 100 first singular values are shown. This case corresponds to a subdiffusive source with reconnection dissipation.

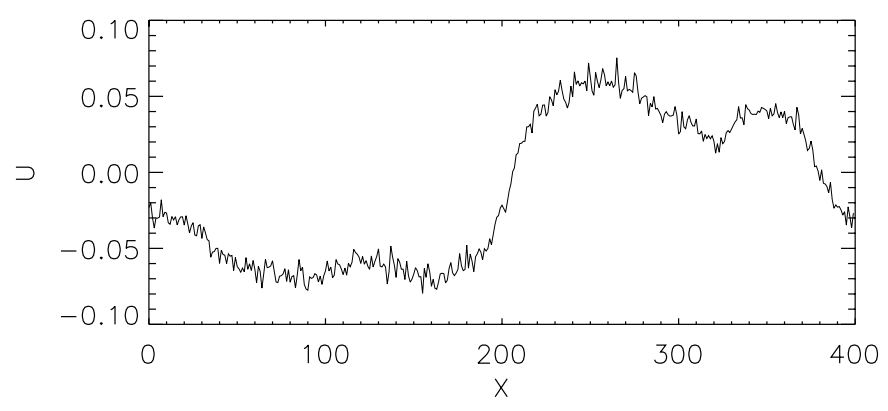

Fig. 7. Spatial profile of the most energetic spatial mode $f_{1}(x)$ obtained by the same Singular Value Decomposition. The data set is the same as in Fig. 6.

\section{Conclusion and discussion}

To study coronal heating due to dissipation of smallscale current layers, we have performed a statistical analysis of a simple model. The model was introduced in Krasnoselskikh et al. (2002), and its principal difference to previous ones is that the system is driven by small-scale homogeneously distributed sources acting on the entire grid for each time step. The idea to consider small-scale sources is motivated by observations by Benz \& Krucker $(1998,1999)$ that heating occurs on the level of the chromosphere, thus, the magnetic field structures, dissipation of which supplies the energy for the heating, are also of a small scale.

The question addressed in this paper is the following: if the actual measurements cannot resolve the characteristic scale of the heating, in what sense are the "macroscopic" observable properties influenced by the properties of the smaller-scale sources?

To answer this question we have carried out a comparative analysis of statistical estimations of the largescale spatial characteristics of the magnetic field such as the correlation length, entropy and most energetic eigenmodes for the different source types that were used in the model (random, chaotic and intermittent with anomalous temporal diffusion).

The "noisy" small scales were filtered out in order to study the large-scale characteristics of the magnetic field. For this purpose we have reconstructed the magnetic
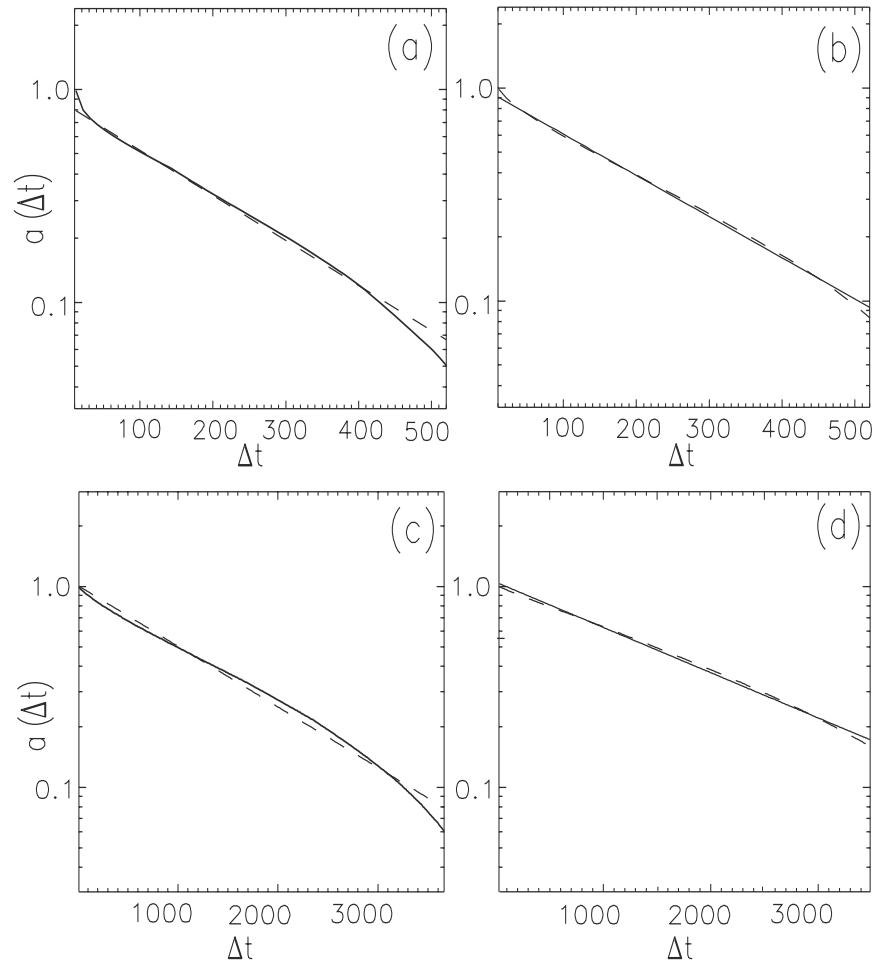

Fig. 8. Averaged temporal correlation function of the magnetic field (solid lines) in log-linear plot calculated from $4 \times 10^{4}$ times steps. Dotted lines represent the best fits by an exponential function. The results are obtained for random and subdiffusive sources, reconnection dissipation, with a threshold of dissipation $j_{\max }=1$. a) Random source, correlation time $\tau \approx 202 ; \mathbf{b})$ the same as previous, but only for the first 20 modes, $\tau \approx 220 ; \mathbf{c}$ ) subdiffusive source, $\tau \approx 1435$; d) the same as c), but only for first 20 modes, $\tau \approx 2958$.

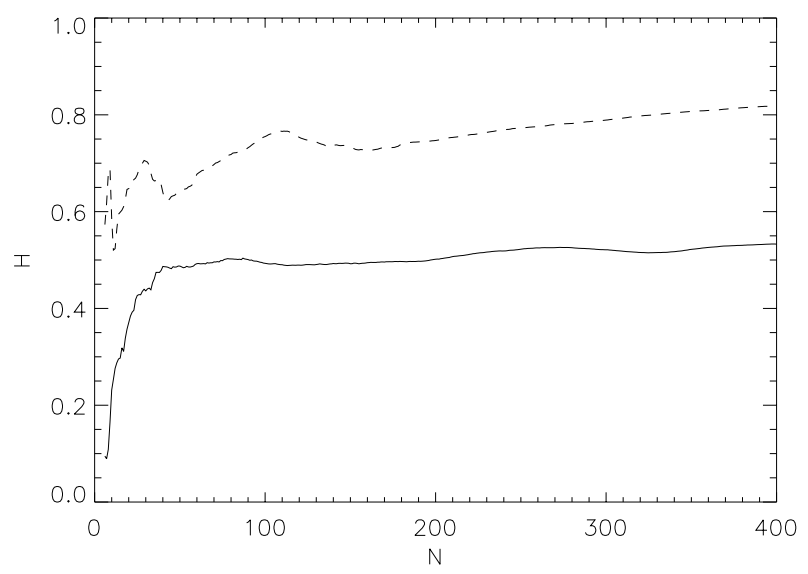

Fig. 9. Partial entropies $H_{M}$ as a function of the grid size $M$. The continuous line is for the subdiffusive source, and the dashed one for the random source. This entropy is normalized so that $H=1$ corresponds to maximum disorder.

field from eigenmodes given by SVD that corresponds to the most energetic coherent structures. The less energetic modes that correspond to the noise level were truncated. 
The results can be summarized as follows:

The large-scale spatial characteristics of the magnetic field such as the correlation length, entropy and most energetic eigenmodes depend significantly on both the statistical properties of small-scale magnetic field sources and the dissipation mechanisms.

- It was found that the temporal average of the correlation function is exponential, i.e. the correlation length is finite and not infinite, as supposed in SOC systems. This length is a little bit larger for the reconnection dissipation and also it depends on the dissipation threshold.

- With the subdiffusive (Geisel) source and reconnection dissipation, the correlation significantly departs from the exponential.

- The Singular Value Decomposition (SVD) allows us to extract the most energetic magnetic field structures, which are essentially larger than the source size and persist for long times, supporting the idea that the plasma can organize on large scales while being driven by small-scale sources.

- Moreover, the entropy computed from the singular values of the magnetic field generated by intermittent sources was found to be much smaller (about 20-30\%) for the subdiffusive source than for other sources. The most intensive in space and long-lived structures are essentially larger in this case also. This indicates a higher level of organization in the system than in the random source case.

The clear difference of the characteristics of spatial complexity in the case of Geisel map sources can be explained in the following way. This deterministic map produces in each cell a random-like diffusion slower than usual (subdiffusion) of magnetic field intensity. On the other hand, the dissipation produces a normal diffusion of the field, i.e. faster magnetic field relaxation along the spatial grid (on average), and relates the temporal properties of the source to spatial properties. This explains why sources with slower diffusion (Geisel) tend to form larger-scale and longer-lived structures than sources with normal diffusion (random, Ulam).

Thus we have shown in the framework of our model that the large-scale spatial structure of the magnetic field in the solar atmosphere also contains important statistical information about the mechanisms of the coronal heating.
Such information can be extracted by SVD-based techniques, which are readily applicable to experimental data and can be used as a complement to the usual analysis of radiated energy.

Acknowledgements. O. Podladchikova is grateful to the French Embassy in Ukraine for the financial support.

\section{References}

Abramenko, V. I., Yurchyshyn, V. B., \& Carbone, V. 1999, in Magnetic fields and Solar Processes, ESA SP-448, 2, 679

Aschwanden, M. J., Tarbell, T. D., Nightingale, R. W., et al. 2000, ApJ, 535, 1047

Aubry, N., Guyonnet, R., \& Lima, R. 1991, J. Stat. Phys., 64, 683

Benz, A. O., \& Krucker, S. 1998, Sol. Phys., 182, 349

Benz, A. O., \& Krucker, S. 1999, A\&A, 341, 286

Dudok de Wit, T. 1995, Plasma Phys. Contr. Fusion, 37, 117

Geisel, T., \& Thomae, S. 1984, Phys. Rev. Lett., 52, 1936

Einaudi, G., \& Velli, M. 1994, in Advances in Sol. Phys., Lecture Notes in Physics, ed. G. Belvedere, M. Rodono, \& G. M. Simnett (Springer, Berlin), 432, 149

Georgoulis, M. K., Vilmer, N., \& Crosby, N. B. 2001, A\&A, 367,326

Golub, G., \& van Loan, C. F. 1996, Matrix Computations, 3rd edition (Johns Hopkins University Press).

Grassberger, P. 1986, Int. J. Theor. Phys., 25, 907

Jensen, H. J. 1998, Self-Organized Criticality (Cambridge University Press)

Isliker, H., Anastasiadis, A., \& Vlahos, L. 2000, A\&A, 363, 1134

Isliker, H., Anastasiadis, A., \& Vlahos, L. 2000, A\&A, in press Krasnoselskikh, V., Podladchikova, O., Lefebvre, B., \& Vilmer, N. 2002, A\&A, 382, 699

Krucker, S., \& Benz, A. O. 1998, ApJ, 501, L213

Lu, E. T., \& Hamilton, R. J. 1991, ApJ, 380, L89

Mitra Kraev, U., \& Benz, A. O. 2001, A\&A, in press

Parker, E. N. 1988, ApJ, 330, 474

Parnell, C. E., \& Jupp, P. E. 2000, ApJ, 529, 554

Podladchikova, O., Krasnoselskikh, V., \& Lefebvre, B. 1999, in Magnetic fields and Solar Processes, ESA SP-448, 1, 553

Priest, E. R., Foley, C. R., Heyvaerts, J., et al. 1998, Nature, 393,545

Priest, E. R., Foley, C. R., Heyvaerts, J., et al. 2000, ApJ, 539, 1002

Vlahos, L., Georgoulis, M., Kluiving, R., et al. 1995, A\&A, 299, 897 